\title{
User Data Control Protocol for Cloud Security
}

\author{
${ }^{* 1}$ Gumpina Babu Rao, ${ }^{2}$ Dr. G. Lavanya Devi, ${ }^{3}$ Prof. N. V. E. S. Murthy \\ ${ }^{1 *}$ Assistant Professor, Department of Computer Science, GITAM (DEEMED TO BE UNIVERSITY), \\ Visakhapatnam, Andhra Pradesh, India \\ ${ }^{2}$ Assistant Professor Department of Computer Science and Systems Engineering Andhra University, \\ Visakhapatnam, Andhra Pradesh, India \\ ${ }^{3}$ Head, Department of Mathematics, J.V.D. College of Science and Technology, Andhra University \\ Visakhapatnam, Andhra Pradesh, India \\ Email: baburaogumpina74@gmail.com
}

Received: $15^{\text {th }}$ October 2018, Accepted: $2^{\text {th }}$ January 2019, Published: $28^{\text {th }}$ February 2019

\begin{abstract}
Fast progression storage advancements, information transformed into prerequisite to standard artefact of a man which needs brisk and ensures isolation during access. Cloud limit therefore created as a appropriate response for area some limitations of getting to and dealing with data at whatever point, wherever what's more, somewhat, in any sum. Particular approaches to manage executing cloud amassing structures have declined or extended customers' trust. This work demonstrates a client-based encryption accumulating structure in which customers control the cryptographic keys process and are allowed to pick exceptional encryption systems, according to their necessities. We in addition make an examination of this framework course of action appeared differently in relation to a couple of features of other equivalent game plans.
\end{abstract}

\section{Keywords}

Cloud Storage Security; Client-Side Encryption; User Controlled Cryptographic Mechanisms

\section{Introduction}

In spite of the way that they pass on new shots and headways to the last customers, there are as yet a couple of issues ought to have been tended to, chiefly in the region of security and information protection and security. Given the relentless prerequisite for administrations change, numerous associations have picked to give these administrations from an outer Cloud stage, be it AWS by Amazon, Azure by Microsoft, Cloud Computing solutions by Google or Cloud Computing solutions by IBM. A development to the complicated cloud enlisting administrations acquired by associations, there are a couple of administrations that singular customer's leverage and are benefitting from, for instance, information stockpiling given by Drive or DropBox or OneDrive by Microsoft. This kind of administrations is likewise alluded to as SaaS and it might be categorized into three models:

A. No Capacity- data is secured as plaintext, without applying any encryption estimations on it. Everything thought of some as, security instruments must be completed, to ensure, at any rate, the uprightness of that data.

B. Host Capacity- data is secured in an encoded edge, yet the cryptographic-keys are made, secured and used by the server. For this kind of limit, the customer can't intrude with the cryptographic keys' lifecycle, thusly an explicit dimension of trust must exist between the customer and the provider.

C. End User Based Capacity- data is secured in encoded outline, while the cryptographic keys are made, secured and controlled on the client application, the cloud having "zero-learning" of the data or of the related cryptographic parts.

To address a part of the issues recognized in many distributed storage structures, various plans have been proposed, for instance, setting up, by the distributed storage providers, an equality between customer security and learning amassed from customers' data [1], realizing a crossbreed encryption and access control scheme that will ensure an occupation based access control [2] or despite depicting a nonexclusive arrangement of activity in which the encryption and deciphering organizations are segregated from capacity advantage [3]. There are similarly extraordinary courses of action proposed starting late, as showed in papers [4-9]. Notwithstanding the way that these courses of action can upgrade the security dimension of a distributed storage system, there is so far a prerequisite for a customer to trust in a provider.

Moreover, our proposed course of action empowers customers to improve through different cryptographic estimations, picking the one that suits their prerequisites, or even implant their own special use of a computation.

\section{Review of the Recent Literatures}

In spite of the way that the a lot of distributed storage providers realizes a server-side encryption show, there are a couple of game plans, existing accessible or proposed in sensible papers, which have structures subject to, to 
an explicit degree, the client side encryption show. When data is transferred to auditors or presented to customers, privacy of audit-relevant data has to be ensured to prevent leakage of sensitive or security-relevant information.[10]. The data owner provides a key to the proxy server using that key proxy is responsible for checking the data.[11] This work firstly evaluates two methods as Tresoritet al.[12] and SpiderOak et al. [13], and further evaluates the remaining models.

A. Tresorit: Tresorit encodes each record on your gadgets previously they are transferred to the cloud applying the Propelled Encryption Standard calculation utilizing 256-piece keys. Your documents never get decoded on Tresorit's servers. Zero-learning security implies that nobody, not by any means Tresorit, can investigate the substance of your documents. Just you and the individuals who you choose to impart to can get to them. Applying a Message Validation Code to each document, Tresorit ensures that the substance of your records can't be adjusted without your insight, regardless of whether someone hacks our framework.

B. SpiderOak: SpiderOak ONE is a super-secure online reinforcement supplier with a large group of highlights more typical to distributed storage, including document match up and sharing. Enhanced evaluating has just expanded its standing. For technophiles searching for a top notch online reinforcement answer for defend their records against defilements, slammed hard drives and lost or stolen PCs, SpiderOak ONE doesn't disillusion. While the sticker price runs higher than contenders like Backblaze, SpiderOak likewise comes outfitted with a few capacities phenomenal in the reinforcement space. The capacity reinforcement boundless PCs as well as match up information crosswise over them, as well, is an immense favorable position when endeavoring to oversee document libraries spread out over numerous machines. In addition, SpiderOak ONE comes outfitted with offer highlights that match those of the best distributed storage contributions. Those advantages, alongside solid security, help rank SpiderOak ONE among the best online reinforcement benefits available today. Continue perusing to discover why Cloudwards.net gives SpiderOak ONE generally high stamps and where we see opportunity to get better.

C. SSeCloud [14]: With the utilization of distributed storage administrations, one of the worries is the means by which to ensure touchy information safely and secretly. While clients appreciate the comfort of information stockpiling given by semi-confided in distributed storage suppliers, they are stood up to with a wide range of dangers in the meantime. In this paper, we present SSeCloud, a protected distributed storage framework that enhances security and ease of use by applying mystery sharing plan to anchor keys. The framework encodes transferring documents on the customer side and parts scrambled keys into three offers. Every one of them is separately put away by clients, distributed storage suppliers and the elective third confided in gathering. Any two of the gatherings can remake keys. Assessment consequences of model framework demonstrate that SSeCloud gives high security without a lot of execution punishment.

D. TwinCloud [15]: TwinCloud is a customer side arrangement giving a safe cloud framework to clients without bargaining the ease of use of cloud sharing. TwinCloud conveys a novel answer for the mind boggling key trade issue and gives a straightforward and functional way to deal with store and offer documents by concealing all the cryptographic and key-appropriation activities from clients. Filling in as a passage, TwinCloud stores the encryption enters and encoded documents in independent mists which facilitate the protected sharing without a requirement for trust to both of the cloud specialist co-ops with

\section{Proposed Framework} the supposition that they don't intrigue with one another.

In this section of the work, the proposed framework is elaborated. The novelty of the proposed framework is to ensure the flexibility of the encryption method to be selected by the client. The proposed framework is elaborated and demonstrated here [Fig -1$]$.

A. Authentication Authority - an untouchable substance that is a considerable and approve relationship to issue and direct open key endorsements for customers.

B. Access Control Servers - this component ensures the access granting or rejection of a customer, in perspective of its capabilities. This module moreover approves the filtering courses of action which associates to the customer information and business functional secrets.

C. Information Storage Servers - this component of the framework gives provides segments to the information to be stored for solitary customers. Since it needn't mess with any incredible handiness, this part can be substituted with organizations from other versatile other storage services from any cloud service providers.

D. Customer Application - this component of the framework ensures the application deployed by the customer can take the advantages of the security protocols deployed by this framework. 


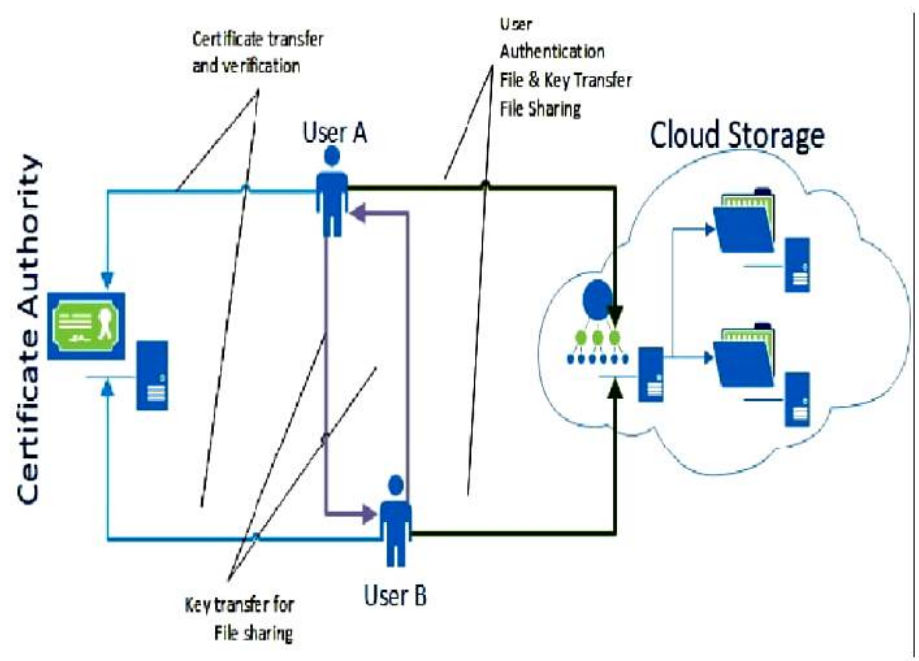

Fig 1. The Proposed Framework

\section{Proposed Algorithm}

A more generic depiction of the workflow of an archive is secured with recouped in this proposed showcase is displayed [Fig - 2] and can be compressed:

Step - 1. The customer sign into the deployed business process with login credentials. After the login process is completed the customer can select the data cluster to be encrypted and can decide on the encryption methodology. Further this encrypted data is to be transmitted over the cloud.

Step - 2. Once the new records are decided to be exchanged, the customer is requested to pick the sort from encryption count (EncAlg) he needs to use. This can be one of the counts given by the application or another, given by the customer as .dll record, composed by specific properties.

Step - 3. At this point the proposed framework makes the required archive encryption key (FEK), using a FIPS 140-2 predictable sporadic number generator joined into the application. Using these FEK and EncAlg, Proposed framework encodes the report and begins the procedure to trade it to the DSS.

Step - 4. Together with the mixed archive, another record, the Association File (AssocFile), is revived with the new information (encoded report name, FEK and EncAlg used), encoded with the all inclusive community key from the customer validation and sent in like manner to the DSS. Thusly, paying little heed to whether the customer has lost the data on his neighborhood drive, he can even now unscramble the information he has secured in the DSS.

Step - 5. While downloading the report, equivalent exercises are executed. Resulting to synchronizing the AssocFile, the Proposed framework downloads the requested report from the DSS, expels the related FEK from the AssocFile, interprets its substance and stores it in a territory picked by the customer on the adjacent drive.

Step - 6. The route toward sharing a record proposes the establishment of more correspondence joins, between a customer An and ACS/DSS, and also between customer An and customer B and CA. These joins are displayed in Figure 3. The central exercises that are executed for this convenience are the going with:

Step - 7. Client A viably sign in Proposed framework and picks the record that he should be shared. The consequent stage is to pick with which customer the record will be granted to, from a summary of customers given by ACS. In the wake of picking the getting party, ACS will give Proposed framework the support of User B that contains its open key (PubKey) and make a channel that will empower User B to see the basic record in its Proposed framework see.

Step - 8. Proposed framework of User A concentrates the FEK and EncAlg related with the shared record and encodes this information using PubKey of User B. He by then either sends it explicitly to User B, if on the web, or sends it to ACS to proper it when User B sign in the structure. 
Step - 9. After adequately tolerating the encoded information, the second user noted as User B can access the encrypted information and further can decide to obtain the decrypted original copy of the information, intended to be received.

Step - 10. While downloading the report, equivalent exercises are executed. Resulting to synchronizing the AssocFile, the Proposed framework downloads the requested report from the DSS, expels the related FEK from the AssocFile, interprets its substance and stores it in a territory picked by the customer on the adjacent drive.

Step - 11. The route toward sharing a record proposes the establishment of more correspondence joins, between a customer An and ACS/DSS, and also between customer An and customer B and CA. These joins are displayed in Figure 3. The central exercises that are executed for this convenience are the going with:

Step - 12. Client A viably sign in Proposed framework and picks the record that he should be shared. The consequent stage is to pick with which customer the record will be granted to, from a summary of customers given by ACS. In the wake of picking the getting party, ACS will give Proposed framework the support of User B that contains its open key (PubKey) and make a channel that will empower User B to see the basic record in its Proposed framework see.

Step - 13. Proposed framework of User A concentrates the FEK and EncAlg related with the shared record and encodes this information using PubKey of User B. He by then either sends it explicitly to User B, if on the web, or sends it to ACS to proper it when User B sign in the structure.

Step - 14. After adequately tolerating the encoded information, the second user noted as User B can access the encrypted information and further can decide to obtain the decrypted original copy of the information, intended to be received.

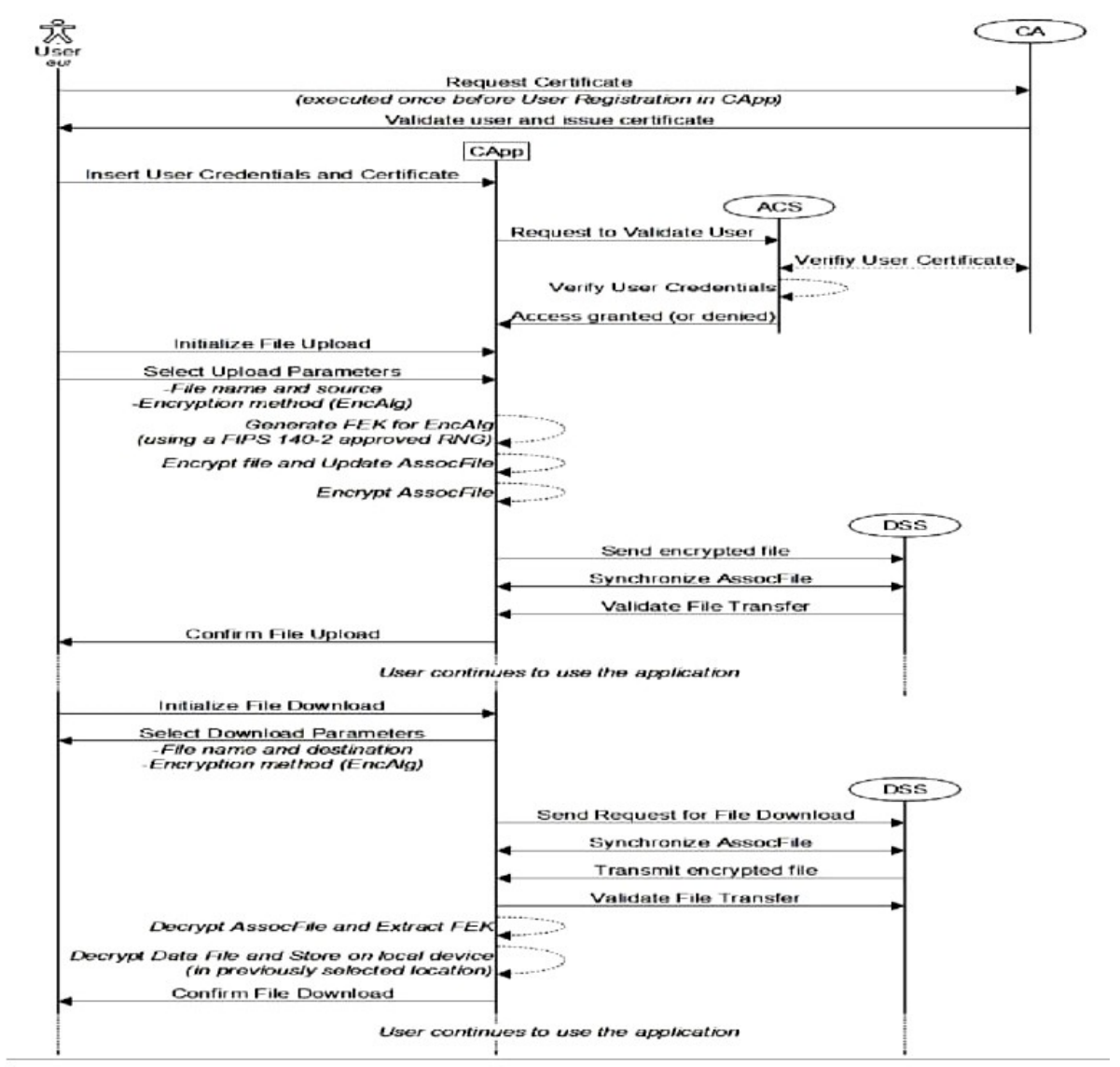

Fig 2. The proposed Algorithm Flow 


\section{Results and Discussion}

From a customer collaboration viewpoint, we assessed a segment of the essential security qualities and how our proposed course of action engages them. As displayed in Table I, our answer securely engages these essential characteristics, while furthermore giving either a clear or an instinctive operational stream for the last customer, dependent upon the choices he takes while securing, recouping or sharing records.

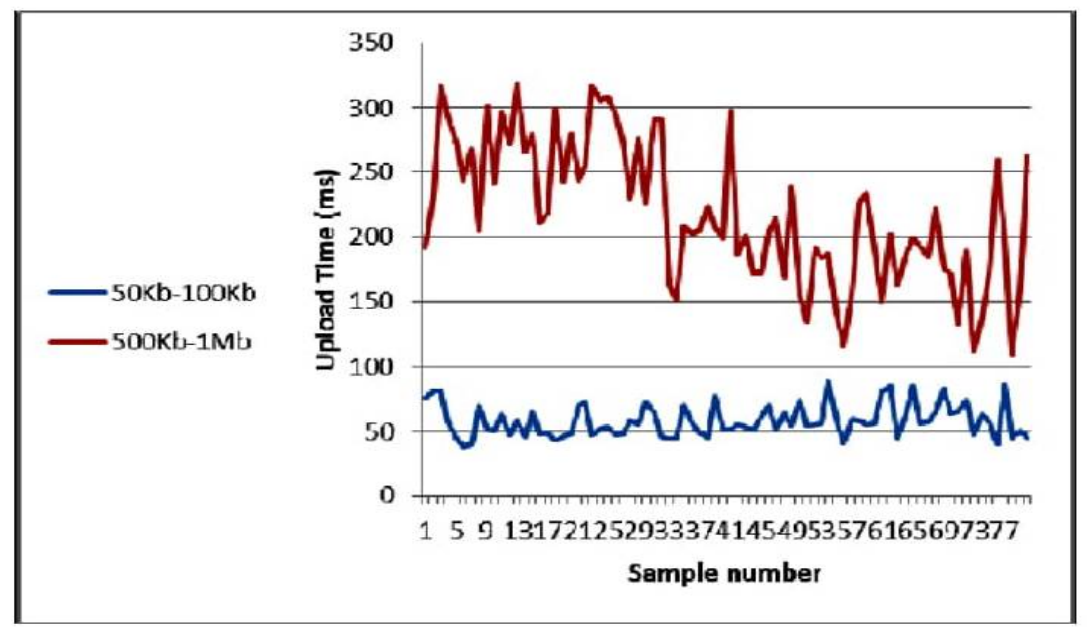

Fig 3. System Performance Analysis with Low Data Loads

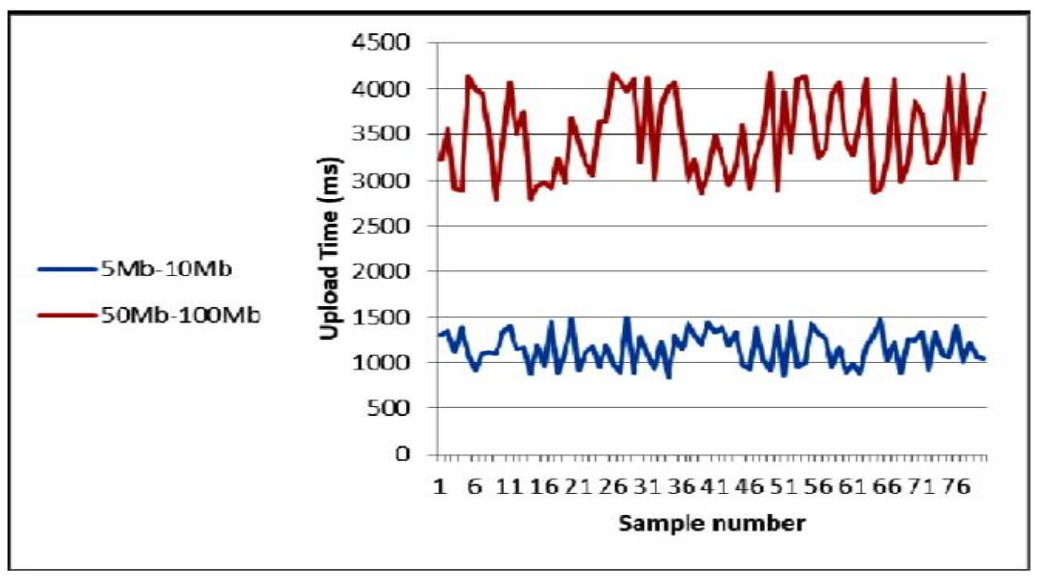

Fig 4. System Performance Analysis with High Data Loads

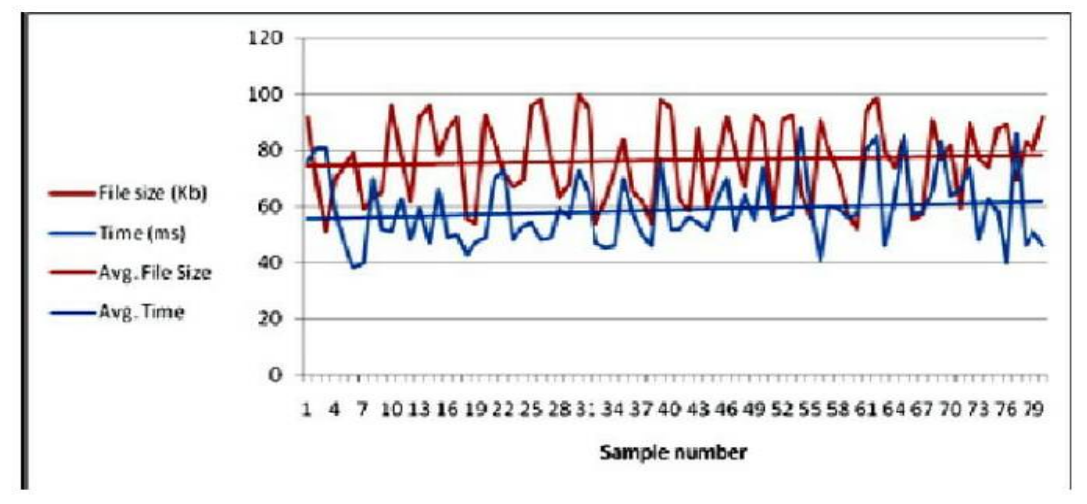

Fig 5. Variation of the Upload Time $(50 \mathrm{~K}-100 \mathrm{~K})$ 


\section{Conclusion}

The developments in the distributed computing and relocation of the current frameworks in to the cloud have constrained the analysts to investigate the cloud security viewpoints. The real test of the cloud security is anchoring the assets on the cloud. The current security strategies can't manage the reality of anchoring the cloud server farm assets as wanted. Accordingly this work gives another strategy for anchoring the cloud assets in a self-practical technique. The proposed structure showed a high exactness of recognizing assaults contrasted with the parallel results of the analysts. The oddity of proposed structure is to share the assault meta data over the protected channel and ended up being the more current element of the exploration for improving the cloud security than previously.

\section{References}

[1] C. Macropoulos, K. M. Martin, "Balacing Privacy and Surveillance in the Cloud", IEEE Cloud Computing, vol. 2, no. 4,2015, pp. 14-21.

[2] L. Zhou, V. Varadharajan, M. Hitchens, "Achieving Secure Role-Based Access Control on Encrypted Data in Cloud Storage", IEEE Transactions on Information Forensics and Security,

[3] J.-J. Hwang, H.-K. Chuang, Y.-C. Hsu, C.-H. Wu, "A Business Model for Cloud Computing Based on a Separate Encryption and Decryption Service", 2011 International Conference on

[4] D. Wilson, J. Avery, Mitigating Data Exfiltration in SaaS Clouds.

[5] M. Mulazzani et al., "Dark Clouds on the Horizon: Using Cloud Storage as Attack Vector and Online Slack Space", USENIX Security Symposium, pp. 65-76, 2011.

[6] V. Rabotla, M. Mannan, "An Evaluation of Recent Secure Deduplication Proposals", Journal of Information Security and Applications, vol. 27, pp. 3-18, 2016.

[7] P. Bhattacharya, T. Q. Phan, L. Liu, "Privacy-preserving Distributed Analytics: Addressing the PrivacyUtility Tradeoff Using Homomorphic Encryption for Peer-to-Peer

[8] M. Grothe, C. Mainka, P. Rösler, J. Jupke, J. Kaiser, J. Schwenk, "Your cloud in my company: Modern rights management services revisited", 2016 11th International Conference on

[9] D. C. Wilson, "Towards Enhancing Security in Cloud Storage Environments", Feb. 2016.

[10] Sebastian Lins, Stephan Schneider, and Ali Sunyaev," Trust is Good, Control is Better: Creating Secure Clouds by Continuous Auditing", IEEE Transactions on Cloud Computing, Vol. 6, No. 3, July-September 2018

[11] Ramesh Patil, Neha Tabassum, "ANCHORING OF CLOUD INFORMATION UNDER KEY

PRESENTATION", International Research Journal of Engineering and Technology (IRJET),volume:05 Issue:08| Aug 2018.

[12 ]. Tresorit, [online] Available: https://tresorit.com.

[13 ]. "SpiderOak", product, [online] Available: https://spideroak.com.

[14 ]. L. Hu, Y. Huang, D. Yang, Y. Zhang, H. Liu, "SSeCloud: Using secret sharing scheme to secure keys", IOP Conference Series: Earth and Environmental Science, vol. 81, 2017.

[15]. K. Bicakci, D. D. Yavuz, S. Gurkan, "TwinCloud: Secure Cloud Sharing Without Explicit Key Management", 2016 IEEE Conference on Communications and Network Security (CNS), 2016. 